False Prophets and Preachers 
Habent sua fata libelli

\title{
Early Modern Studies Series
}

\author{
General Editor \\ Michael Wolfe \\ Queens College, CUNY
}

Editorial Board of Early Modern Studies

Elaine Beilin

Framingham State College

Christopher Celenza

Johns Hopkins University

Barbara B. Diefendorf

Boston University

Paula Findlen

Stanford University

Scott H. Hendrix

Princeton Theological Seminary

Jane Campbell Hutchison

University of Wisconsin-Madison

Mary B. McKinley

University of Virginia
Raymond A. Mentzer

University of Iowa

Robert V. Schnucker

Truman State University, Emeritus

Nicholas Terpstra

University of Toronto

Margo Todd

University of Pennsylvania

James Tracy

University of Minnesota

Merry Wiesner-Hanks

University of WisconsinMilwaukee 


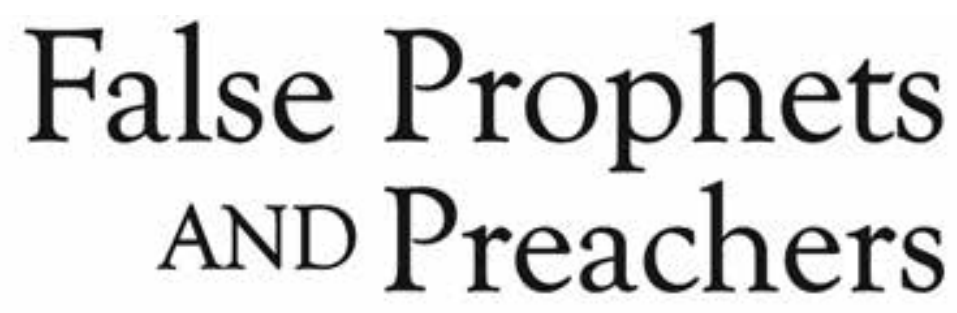

Henry Gresbeck's Account of the Anabaptist Kingdom of Münster CHRISTOPHER S. MACKAY

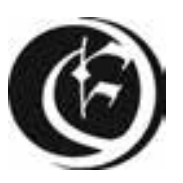

Early Modern Studies 18

Truman State University Press

Kirksville, Missouri 
Copyright (C) 2016 Truman State University Press, Kirksville, Missouri, 63501

All rights reserved

tsup.truman.edu

Cover art: Johann Karl Ulrich Bähr, Jan van Leiden tauft ein Mädchen. Oil on canvas, 1840. Westphalian State Museum of Art and Cultural History.

Cover design: Teresa Wheeler

Library of Congress Cataloging-in-Publication Data

Names: Gresbeck, Heinrich, active 1540. | Mackay, Christopher S., 1962-

Title: False prophets and preachers : Henry Gresbeck's account of the

Anabaptist kingdom of Münster / [translated and annotated by] Christopher

Mackay.

Other titles: Berichte der Augenzeugen über das münsterische

Wiedertäuferreich. English

Description: Kirksville, Missouri : Truman State University Press, 2016. |

Series: Early modern studies ; $18 \mid$ Includes bibliographical references.

Identifiers: LCCN 2015042883 (print) | LCCN 2016006745 (ebook) | ISBN

9781612481418 (library binding : alkaline paper) | ISBN 9781612481425 ()

Subjects: LCSH: Gresbeck, Heinrich, active 1540.|

Anabaptists-Germany-Münster in Westfalen-History-16th century. |

Münster in Westfalen (Germany)_Church history-16th century. | Münster

in Westfalen (Germany)—History-16th century. |

Germany-History-1517-1648.

Classification: LCC BX4933.G3 G7413 2016 (print) | LCC BX4933.G3 (ebook) |

DDC $943 / .5614031-\mathrm{dc} 23$

LC record available at http://lccn.loc.gov/2015042883

No part of this work may be reproduced or transmitted in any format by any means without written permission from the publisher.

The paper in this publication meets or exceeds the minimum requirements of the American National Standard for Information Sciences-Permanence of Paper for Printed Library Materials, ANSI Z39.48-1992. 
Kelliae carissimae ac de me optime merenti, cuius ocelli, siquid desit meis, supplent! 
